\title{
Can energetic vaccines, based on physics, be the sound options for COVID-19 and other pandemics in the absence of pharmaceutical vaccines?
}

\author{
Savely Yurkovsky ${ }^{1}$ \\ ${ }^{1}$ Affiliation not available
}

November 17, 2020

\begin{abstract}
The proposed approach presents substantial scientific evidence based on physics and clinical data to support a clinical trial of an energetic SARS-CoV-2 vaccine. This is particularly imperative in the absence of a pharmaceutical vaccine as well as its uncertain future efficacy and safety, considering the limited time for testing and rapid mutations of the virus. Some of the vaccine trials have already been halted due to volunteers falling ill. Physics and biophysics of the fundamental electromagnetic nature of the living matter, including microbes as well as water, support the imprinting of electromagnetic fields of microbes in water that elicits an immune response. Energetic vaccines can be rapidly mass-produced for both prophylaxis and treatment of the public and healthcare personnel against the original as well as mutated viral strains. These can be prepared through a standard homeopathic process, as well as an energy field imprinting device, ${ }^{*}$ offering a unique potential in versatility, speed, and low cost in mass protection against the current and future public health emergencies. Other viral infections, epidemics, antibiotic-resistant, and vector-borne infections could be similarly addressed. Paradoxically, in spite of a general perception of homeopathy by physicians as the most alien and least scientific, among complementary and alternative medicine (CAM), it is just the opposite. Homeopathy carries formidable scientific support, with the specific proposed homeopathic model being fully in line with physicians' superior knowledge of infectious diseases and medicine, making its adaptation into their practice intuitive and more effective than in the hands of non-MDs or DOs homeopaths. The practical advantage of the proposed homeopathic model in acute infections versus its oldest one of multisystemic, totality approach, yielding mixed results in chronic diseases, parallels a similar prevailing success of the corresponding conventional modalities in acute versus chronic diseases.
\end{abstract}

\section{Hosted file}

IJCP MAIN DOCUMENT.pdf available at https://authorea.com/users/376559/articles/493484can-energetic-vaccines-based-on-physics-be-the-sound-options-for-covid-19-and-otherpandemics-in-the-absence-of-pharmaceutical-vaccines 